\title{
Time-dependent $\boldsymbol{V}$-representability on lattice systems
}

\author{
Yonghui Li and C. A. Ullrich ${ }^{\text {a) }}$ \\ Department of Physics and Astronomy, University of Missouri, Columbia, Missouri 65211, USA
}

(Received 19 April 2008; accepted 13 June 2008; published online 25 July 2008)

\begin{abstract}
We study the mapping between time-dependent densities and potentials for noninteracting electronic systems on lattices. As discovered recently by Baer [J. Chem. Phys. 128, 044103 (2008)], there exist well-behaved time-dependent density functions on lattices which cannot be associated with any real time-dependent potential. This breakdown of time-dependent $V$-representability can be tracked down to problems with the continuity equation which arise from discretization of the kinetic-energy operator. Examples are given for lattices with two points and with $N$ points, and implications for practical numerical applications of time-dependent density-functional theory are discussed. In the continuum limit, time-dependent noninteracting $V$-representability is restored. (C) 2008 American Institute of Physics. [DOI: 10.1063/1.2955733]
\end{abstract}

\section{INTRODUCTION}

Time-dependent density-functional theory ${ }^{1}$ (TDDFT) is a widely used methodology for the description of electron dynamics in atoms, molecules, and solids. The birth of TDDFT dates back to the seminal work by Runge and Gross ${ }^{2}$ (RG) who proved a one-to-one correspondence between time-dependent densities $n(\mathbf{r}, t)$ and time-dependent potentials $V(\mathbf{r}, t)$. The RG theorem ${ }^{2}$ can be viewed as the timedependent analog of the Hohenberg-Kohn (HK) theorem ${ }^{3}$ in static DFT. ${ }^{4}$

While TDDFT has found widespread practical use for describing such diverse phenomena as molecular excitations, nanoscale electron transport, or strong-field processes, there is a continuing need to study the fundamental underpinnings of the theory. Some of the basic issues in TDDFT that have recently received much attention are nonadiabaticity and memory effects ${ }^{5,6}$ and causality and the time-dependent variational principle. $^{7}$

This paper has been motivated by an unexpected and disquieting discovery made recently by Baer: ${ }^{8}$ on lattice systems there exist seemingly well-behaved time-dependent density functions which are not $V$-representable (VR), i.e., which cannot be associated with any time-dependent potential. Attempts to invert the time-dependent Schrödinger equation (TDSE) to explicitly construct the potential from such densities encounter severe numerical instabilities. It was suggested in Ref. 8 that such instabilities might be potentially disastrous for practical applications of TDDFT, since they could cause the exchange-correlation $(\mathrm{XC})$ potential $V_{\mathrm{XC}}$ to become an extremely sensitive functional of the density.

The purpose of this paper is to shed light on two important questions which are raised by Baer's study: (1) what is the fundamental reason for the absence of time-dependent $V$-representability on lattices and (2) how does timedependent $V$-representability emerge in the continuum limit? Both questions have a profound impact on our fundamental understanding of TDDFT as well as on practical applications.

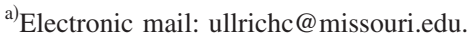

We will give some general answers, as well as some specific illustrations for the mapping between time-dependent potentials and noninteracting densities on one-dimensional lattices.

\section{DFT ON LATTICE SPACES}

The basic existence theorem of ground-state DFT, the (first) HK theorem, ${ }^{3}$ states that there is a $1: 1$ correspondence between external potentials and ground-state densities. In other words, it cannot happen that two potentials that differ by more than a constant produce the same ground-state density of an $N$-particle system. The proof of this theorem is based on the Ritz variational principle,

$$
\left\langle\Psi_{\mathrm{gs}}|\hat{H}| \Psi_{\mathrm{gs}}\right\rangle<\langle\Psi|\hat{H}| \Psi\rangle,
$$

where $\hat{H}$ is the Hamiltonian of the interacting many-particle system, $\Psi_{\mathrm{gs}}$ is the associated ground-state wave function, and $\Psi$ is any other many-particle wave function. Notice that this fundamental variational principle does not require that the system lives in a continuous coordinate space; it remains valid for systems that are defined on a discrete space, such as on a real-space lattice or using a finite set of basis functions.

The basic DFT framework assumes interacting and noninteracting $V$-representabilities. ${ }^{9}$ In other words, it is assumed that any density function which is mathematically reasonable (i.e., positive valued, not diverging, and integrating to $N$ ) is a ground-state density belonging to some external potential, for a given form of the electron-electron interaction. This property is of obvious importance for a mathematically meaningful implementation of variations with respect to the density. In practice, interacting $V$-representability is a less critical issue, since one is only interested in densities that can be realized in nature as physical densities of systems of interacting electrons. The goal of Kohn-Sham DFT is to represent these densities via auxiliary, noninteracting systems, which obviously requires them to be noninteracting VR, and surrounded by VR "neighborhoods" in the space of density functions. We shall therefore focus exclusively on noninteracting $V$-representability. Unfortu- 
nately, attempts to prove $V$-representability for all densities in the continuum case (e.g., in Ref. 10) have so far not been fully successful.

In ground-state DFT, lattice systems have been much used to address the $V$-representability problem. In 1983, Kohn ${ }^{11}$ showed that in the vector space of density functions that are defined on discrete lattices, a density in the neighborhood of a VR density is also VR. In 1985, Chayes et al. ${ }^{12}$ proved that any mathematically well-behaved density function on finite or infinite lattices can be represented as the density of a pure ground state or as an ensemble density associated with a degenerate ground state. In density vector spaces of arbitrary dimension, it is equally likely to encounter pure-state and ensemble-state VR densities. ${ }^{13}$ Since the lattice can be arbitrarily dense, this effectively solves the $V$-representability problem in DFT. In a related approach, $V$-representability was recently proven by Lammert ${ }^{14}$ for coarse-grained systems.

We mention that lattice systems have also been very helpful in studying the issue of nonuniqueness in spin-DFT, where it was discovered ${ }^{15}$ that there are several different types of situations where the mapping between densities and spin magnetizations $\{n(\mathbf{r}), \mathbf{m}(\mathbf{r})\}$ and potentials and magnetic fields $\{V(\mathbf{r}), \mathbf{B}(\mathbf{r})\}$ is not unique. In the continuum limit, only some special cases of nonuniqueness in spin-DFT survive.

\section{V-REPRESENTABILITY IN CONTINUOUS- AND DISCRETE-SPACE TDDFT}

The issue of $V$-representability in TDDFT turns out to be quite different from static DFT. Van Leeuwen ${ }^{16}(\mathrm{vL})$ showed that, assuming that the initial state is noninteracting VR, there is always a unique time-dependent potential $V(\mathbf{r}, t)$ in a noninteracting system that produces a given interacting density $n(\mathbf{r}, t)$ at all times. In other words, the time-dependent $V$-representability problem in the continuum case can be considered solved by the vL construction. In view of this, the finding by $\mathrm{Baer}^{8}$ that time-dependent noninteracting $V$-representability breaks down on lattices is particularly unsettling and calls for an explanation.

Unlike the HK theorem in static DFT, the RG theorem ${ }^{2}$ and the $\mathrm{vL}$ construction ${ }^{16}$ are not based on a minimum principle. Instead, it is demonstrated that potentials which differ from each other by more than a time-dependent function cause the time evolution of the respective systems to proceed in such a manner that the time-dependent densities are not the same. The original RG proof requires two steps: first it is shown that if a system evolves from a given initial state under the influence of two external potentials $V(\mathbf{r}, t)$ and $V^{\prime}(\mathbf{r}, t) \neq V(\mathbf{r}, t)+c(t)$, then the current densities $\mathbf{j}(\mathbf{r}, t)$ and $\mathbf{j}^{\prime}(\mathbf{r}, t)$ are different. The next step shows by virtue of the continuity equation that the associated particle densities $n(\mathbf{r}, t)$ and $n^{\prime}(\mathbf{r}, t)$ become different, too. The continuity equation also plays a crucial role in the vL construction.

Let us therefore take a closer look at the continuity equation, given by

$$
\dot{n}(\mathbf{r}, t)=-\nabla \cdot \mathbf{j}(\mathbf{r}, t),
$$

where the dot denotes a partial derivative with respect to time. Here and in the following, we use Hartree atomic units with $\hbar=e=m=1$. For the simplest case, a single particle in one dimension, the continuity equation, becomes

$$
\dot{n}(x, t)=\frac{i}{2}\left[\psi^{*}(x, t) \frac{\partial^{2} \psi(x, t)}{\partial x^{2}}-\psi(x, t) \frac{\partial^{2} \psi^{*}(x, t)}{\partial x^{2}}\right],
$$

which follows from the TDSE by assuming that the potential is local and real.

Let us now treat the time variable as continuous and the space variable $x$ as discretized on a grid with equidistant grid spacing $a$. The position of the $j$ th lattice point is $x_{j}$, and $n_{j}$ denotes the number of particles in a bin of width $a$, centered at $x_{j}$. Thus, the lattice quantity corresponding to the particle density $n(x, t)$ is $n_{j}(t) / a$. The advantage of this definition is that the normalization condition on an $N$-point lattice becomes simply

$$
\sum_{j=1}^{N} n_{j}=1
$$

For the wave functions, we have $\psi(x, t) \rightarrow \psi_{j}(t) / \sqrt{a}$.

Using a three-point finite-difference representation of the Laplacian operator (which is accurate to within terms of or$\left.\operatorname{der} a^{2}\right),{ }^{17}$ we have

$$
\dot{n}_{j}=\frac{i}{2 a^{2}} \psi_{j}^{*}\left[\psi_{j+1}-2 \psi_{j}+\psi_{j-1}\right]+\text { c.c. },
$$

where the time arguments are suppressed for brevity. Without loss of generality we take the following form of the wave function:

$$
\psi_{j}=\sqrt{n_{j}} e^{i \alpha_{j}}
$$

where $\alpha_{j}$ is a real-valued time-dependent phase. The discretized continuity equation then becomes

$$
\dot{n}_{j}=\frac{1}{a^{2}} \sqrt{n_{j} n_{j+1}} \sin \left(\alpha_{j}-\alpha_{j+1}\right)+\frac{1}{a^{2}} \sqrt{n_{j} n_{j-1}} \sin \left(\alpha_{j}-\alpha_{j-1}\right) .
$$

We will now show that $\dot{n}_{j}$ is not allowed to take on arbitrary values. We have

$$
\left|\dot{n}_{j}\right| \leq \frac{1}{a^{2}}\left[\sqrt{n_{j} n_{j+1}}+\sqrt{n_{j} n_{j-1}}\right] .
$$

Next, let $n_{j+1}=n_{j}+\Delta_{j}$. Using the normalization condition (4) one obtains $n_{j-1} \leq 1-2 n_{j}-\Delta_{j}$ (the equal sign arises for lattices consisting only of three points or if the potential is infinity on all but the three points $j-1, j$, and $j+1)$. Thus,

$$
\left|\dot{n}_{j}\right| \leq \frac{\sqrt{n_{j}}}{a^{2}}\left[\sqrt{n_{j}+\Delta_{j}}+\sqrt{1-2 n_{j}-\Delta_{j}}\right] .
$$

The right-hand side is maximized for $\Delta_{j}=-1 / 4$ and $n_{j}=1 / 2$, which leads to 


$$
\left|\dot{n}_{j}\right| \leq \frac{1}{\sqrt{2} a^{2}} .
$$

On a one-dimensional equidistant grid with a three-point finite-difference representation of the kinetic-energy operator, the time derivative of the density is restricted by the local upper bound (10). In other words, the density on a given grid point cannot change arbitrarily fast.

The upper bound (10) can be generalized for a $d$-dimensional grid using a $k$-point formula for the Laplacian operator, ${ }^{17}$ resulting in $\left|\dot{n}_{j}\right| \leq A_{k}^{d} / a^{2}$, where $A_{k}^{d}$ is a finite numerical constant. It is easy to see that on an equidistant $d$-dimensional grid, $A_{k}^{d}=d A_{k}^{1}$. Going beyond three-point formulas, one finds, for instance, $A_{5}^{d}=(4 / 3) A_{3}^{d}$ for five-point formulas and $A_{k}^{d} \rightarrow 2 A_{3}^{d}$ for large $k$.

Thus, there are fundamental limits, determined by the grid spacing, the number of dimensions, and the discrete representation of the kinetic-energy operator, on how fast lattice densities can locally change without violating the continuity equation. The RG theorem and the $\mathrm{vL}$ construction are thus restricted to time-dependent densities which satisfy these bounds. Otherwise, time-dependent $V$-representability cannot be guaranteed. We will now illustrate this with some explicit examples.

\section{THE TWO-POINT LATTICE}

The case of the two-point lattice was already treated by Baer; ${ }^{8}$ nevertheless, it is a very instructive and pedagogical model, and we will discuss it here in some detail.

We start with the single-electron TDSE in real space,

$$
i \frac{\partial}{\partial t} \psi(\mathbf{r}, t)=\left[-\frac{\nabla^{2}}{2}+\tilde{V}(\mathbf{r}, t)\right] \psi(\mathbf{r}, t) .
$$

On a two-point lattice, the wave function is completely specified by its values on point 1 and on point $2, \psi_{1}(t)$ and $\psi_{2}(t)$. These two (complex) quantities can be arranged as the components of a vector. The TDSE can then be discretized and written in the form of a $2 \times 2$ matrix equation,

$$
i \frac{\partial}{\partial t}\left(\begin{array}{l}
\psi_{1}(t) \\
\psi_{2}(t)
\end{array}\right)=\left(\begin{array}{cc}
\frac{1}{a^{2}}+\tilde{V}_{1}(t) & -\frac{1}{2 a^{2}} \\
-\frac{1}{2 a^{2}} & \frac{1}{a^{2}}+\tilde{V}_{2}(t)
\end{array}\right)\left(\begin{array}{l}
\psi_{1}(t) \\
\psi_{2}(t)
\end{array}\right)
$$

where we used a standard three-point finite-difference representation of the kinetic-energy operator assuming that the two lattice points have a spacing $a$.

The notation can be simplified recognizing that an overall constant shift of the potential is irrelevant. This means that the potential can be redefined as $V_{j}=1 / a^{2}+\tilde{V}_{j}, j=1,2$, and

$$
i \frac{\partial}{\partial t}\left(\begin{array}{l}
\psi_{1}(t) \\
\psi_{2}(t)
\end{array}\right)=\left(\begin{array}{cc}
V_{1}(t) & -\frac{1}{2 a^{2}} \\
-\frac{1}{2 a^{2}} & V_{2}(t)
\end{array}\right)\left(\begin{array}{l}
\psi_{1}(t) \\
\psi_{2}(t)
\end{array}\right)
$$

We now write the wave function on points 1 and 2 in the form (6). The densities and phases, $n_{1,2}$ and $\alpha_{1,2}$, are real

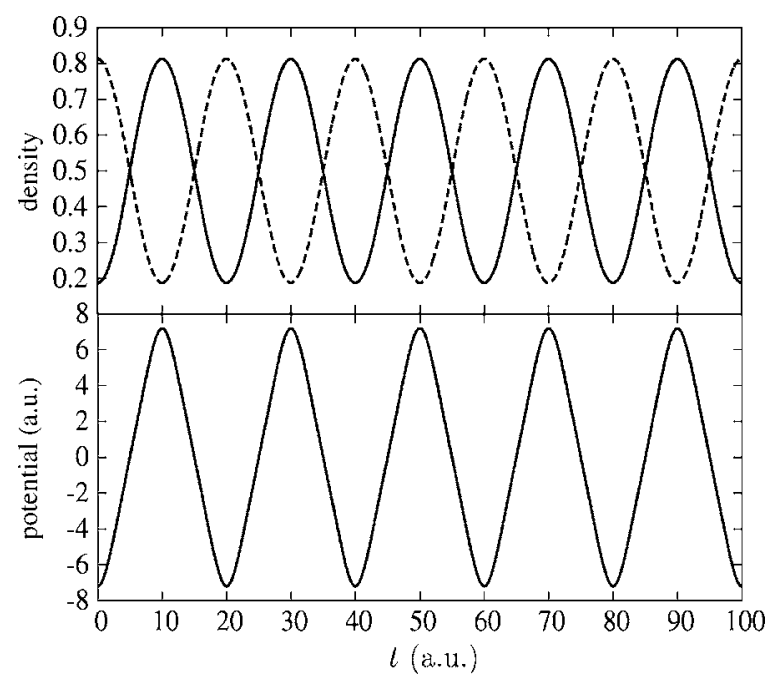

FIG. 1. Top: two-point lattice densities $n_{1}$ (full line) and $n_{2}$ (dashed line) resulting from $n(x, t)$ of Eq. (21), with $\omega=0.1 \pi$. Bottom: potential difference $\Delta V=V_{2}-V_{1}[$ Eq. (18)].

functions of time. Inserting this in the TDSE (13) gives the following equations for the real and imaginary parts of the potential on point 1 ,

$$
\begin{aligned}
& \Re V_{1}=-\dot{\alpha}_{1}+\frac{1}{2 a^{2}} \sqrt{\frac{n_{2}}{n_{1}}} \cos \left(\alpha_{2}-\alpha_{1}\right), \\
& \Im V_{1}=\frac{\dot{n}_{1}}{2 n_{1}}+\frac{1}{2 a^{2}} \sqrt{\frac{n_{2}}{n_{1}}} \sin \left(\alpha_{2}-\alpha_{1}\right),
\end{aligned}
$$

and similar on point 2 . If we require the potential to be purely real, then the imaginary part, given by Eq. (15), vanishes. We can then solve for the phase difference,

$$
\alpha_{1}-\alpha_{2}=\sin ^{-1}\left(\frac{a^{2} \dot{n}_{1}}{\sqrt{n_{1} n_{2}}}\right) .
$$

Due to norm conservation (4), we have only one independent density variable, since $n_{1}=1-n_{2}$. Likewise, there is only one independent potential variable, since an arbitrary overall constant shift only affects the phases. ${ }^{2}$ Thus, only the potential difference matters, and we let $\Delta V \equiv \mathfrak{R} V_{2}-\mathfrak{R} V_{1}$. From Eq. (14) we get

$$
\Delta V=-\left(\dot{\alpha}_{2}-\dot{\alpha}_{1}\right)+\frac{1}{2 a^{2}}\left[\sqrt{\frac{n_{1}}{n_{2}}}-\sqrt{\frac{n_{2}}{n_{1}}}\right] \cos \left(\alpha_{1}-\alpha_{2}\right),
$$

and the phase differences can be eliminated using Eq. (16). After some algebra, this yields the potential difference as a function of the densities at points 1 and 2,

$$
\Delta V=\frac{a^{2}}{\sqrt{n_{1} n_{2}-a^{4} \dot{n}_{1}^{2}}}\left(\ddot{n}_{1}+\frac{n_{1}-n_{2}}{2 a^{4}}\right) \text {. }
$$

This equation for $\Delta V$ is well behaved and has a unique solution as long as $n_{1} n_{2}-a^{4} \dot{n}_{1}^{2}>0$ or 


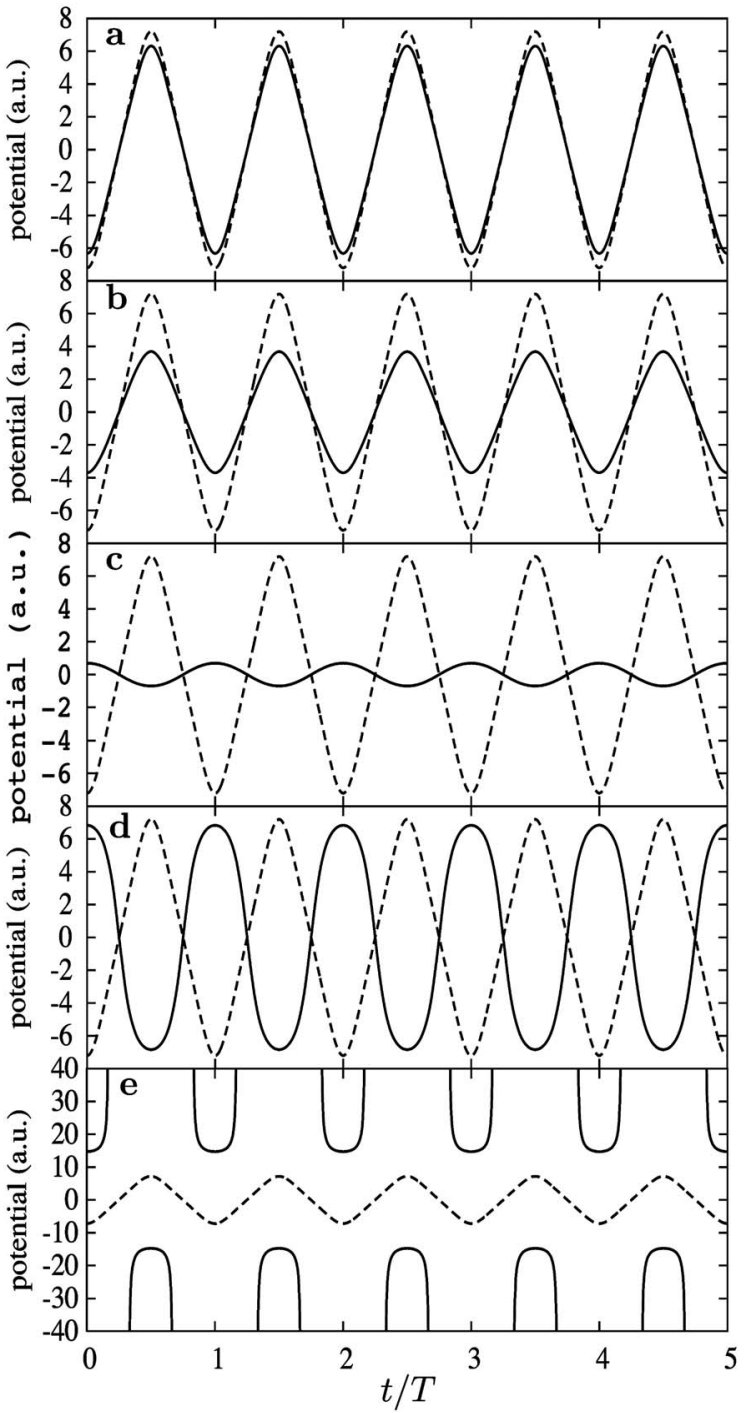

FIG. 2. Full lines: potential difference $\Delta V=V_{2}-V_{1}$ for $\omega=\pi, 2 \pi, 3 \pi, 4 \pi$, and $5 \pi[(\mathrm{a})-(\mathrm{e})]$, with $T=2 \pi / \omega$. Dashed lines: adiabatic potential [Eq. (22)]. Panel (e) shows regions where no potential exists that produces the given lattice densities $n_{1}(t)$ and $n_{2}(t)$, which means that they are non-VR.

$$
\left|\dot{n}_{1,2}\right|<\frac{\sqrt{n_{1} n_{2}}}{a^{2}}
$$

( $n_{1}$ and $n_{2}$ themselves are of course always $>0$; furthermore, $\dot{n}_{1}=-\dot{n}_{2}$ ). This yields the following upper bound:

$$
\left|\dot{n}_{1,2}\right|_{\max }=\frac{1}{2 a^{2}} .
$$

Time-dependent single-particle lattice densities $n_{1}, n_{2}$ which violate conditions (19) or (20) at any time $t$ do not produce a real value of $\Delta V$ at that time and are therefore not timedependent VR. This upper bound for the two-point lattice is clearly more restrictive than condition (10) for a general onedimensional lattice.

As an illustration, let us consider a one-dimensional time-dependent density function of the form

$$
\begin{aligned}
n(x, t)= & A_{1}^{2} \cos ^{2}(\pi x)+A_{2}^{2} \sin ^{2}(2 \pi x) \\
& +2 A_{1} A_{2} \cos (\pi x) \sin (2 \pi x) \cos (\omega t),
\end{aligned}
$$

$-1 / 2 \leq x \leq 1 / 2$, arising from a superposition of the first and second single-particle eigenstates of a particle in a onedimensional box of length 1 . We discretize $n(x, t)$ on a twopoint lattice such that $n_{1,2}=n\left(x_{1,2}, t\right)$, where $x_{1}=-1 / 6$ and $x_{2}=1 / 6$, and the lattice spacing is $a=1 / 3$. The lattice normalization condition (4) requires choosing $A_{1}$ and $A_{2}$ such that $n_{1}+n_{2}=1$.

The top panel of Fig. 1 shows $n_{1}$ and $n_{2}$ as a function of time for a frequency $\omega=0.1 \pi$ and $A_{1}=0.77$ and $A_{2}=0.27$, together with the associated potential difference $\Delta V$, following from Eq. (18). This is a case where the lattice densities are VR, and the potential is a well-behaved function of time which drives the particle density periodically from the first to the second lattice point and back. In fact, in this lowfrequency case the adiabatic approximation for the potential,

$$
\Delta V_{\text {adia }}(t)=\frac{n_{1}(t)-n_{2}(t)}{2 a^{2} \sqrt{n_{1}(t) n_{2}(t)}}
$$

(which assumes that at each moment in time the system is in the ground state associated with the instantaneous potential), is indistinguishable from the exact $\Delta V(t)$.

Figure 2 shows the potential reproducing $n_{1}$ and $n_{2}$ at higher frequencies, from $\omega=\pi$ through $5 \pi$. As $\omega$ grows, the time-dependent potential $\Delta V$ starts to differ more and more from the adiabatic approximation $\Delta V_{\text {adia. }}$ In fact, there is a crossover at $\omega=2.865 \pi$ where one finds $\Delta V(t) \equiv 0$, which corresponds to free charge-density oscillations on the twopoint lattice with frequency $\omega=1 / a^{2}$ (the difference of the two eigenvalues of the static Schrödinger equation with $V_{1}$ $\left.=V_{2}=0\right)$. For $\omega>2.865 \pi, \Delta V$ and $\Delta V_{\text {adia }}$ are out of phase, which may be viewed as an indication of entering the highfrequency regime.

For $\omega>4.587 \pi$ one finds that $\Delta V$ diverges periodically, and there are regions in time where there is no solution at all, as seen for $\omega=5 \pi$ in panel (e) of Fig. 2. This is a striking example where the time-dependent lattice density is non-VR.

In the regions in which $\Delta V$ does not exist, condition (19) is violated, which implies that the densities change too rapidly. In other words, there is a limit on how much density can move how fast between the two points. Violation of this limit means that no real-valued time-dependent lattice potential can be found which is capable of driving the system strongly and fast enough to produce the given time-dependent density.

To conclude this section, we mention that some of the issues addressed here have also been recently discussed by Verdozzi in the context of Hubbard dimers. ${ }^{18}$

\section{THE N-POINT LATTICE}

For a linear $\mathrm{N}$-point lattice with equidistant grid spacing $a$ and a three-point finite-difference formula, the discretized TDSE reads 


$$
i\left(\begin{array}{c}
\dot{\psi}_{1} \\
\dot{\psi}_{2} \\
\dot{\psi}_{3} \\
\vdots \\
\dot{\psi}_{N}
\end{array}\right)=\left(\begin{array}{ccccc}
V_{1} & -\frac{1}{2 a^{2}} & 0 & \ldots & 0 \\
-\frac{1}{2 a^{2}} & V_{2} & -\frac{1}{2 a^{2}} & \ldots & 0 \\
0 & -\frac{1}{2 a^{2}} & V_{3} & \ldots & 0 \\
\vdots & \vdots & \vdots & \ddots & \vdots \\
0 & 0 & 0 & \ldots & V_{N}
\end{array}\right)\left(\begin{array}{c}
\psi_{1} \\
\psi_{2} \\
\psi_{3} \\
\vdots \\
\psi_{N}
\end{array}\right),
$$

where the time arguments of the $\psi_{i}$ and $V_{i}$ have been omitted and the constant $1 / a^{2}$ coming from the finite-difference kinetic-energy operator has been absorbed in the potentials $V_{i}(t)$, as in Eq. (13).

It is straightforward to generalize the analysis of the previous section to the $\mathrm{N}$-point lattice. We again use ansatz (6) and eliminate the phases using the requirement that the potential be real at each point. Defining

$$
S_{k}=\sum_{j=1}^{k} \dot{n}_{j}
$$

we obtain after some algebra the following expression for the point-to-point potential differences:

$$
\begin{aligned}
V_{k+1}-V_{k}= & \frac{a^{2}}{\sqrt{n_{k} n_{k+1}-a^{4} S_{k}^{2}}}\left[\dot{S}_{k}-\frac{\left(n_{k} \dot{n}_{k+1}+n_{k+1} \dot{n}_{k}\right) S_{k}}{2 n_{k} n_{k+1}}\right] \\
& +\frac{n_{k}-n_{k+1}}{2 a^{2} n_{k} n_{k+1}} \sqrt{n_{k} n_{k+1}-a^{4} S_{k}^{2}} \\
& +\frac{1}{2 a^{2} n_{k+1}} \sqrt{n_{k+1} n_{k+2}-a^{4} S_{k+1}^{2}} \\
& -\frac{1}{2 a^{2} n_{k}} \sqrt{n_{k-1} n_{k}-a^{4} S_{k-1}^{2}}, \quad k=1, \ldots, N-1 .
\end{aligned}
$$

For notational convenience, Eq. (25) makes reference to the zeroth and the $(N+1)$ st lattice point. These points should be regarded as "virtual points," consistent with the boundary condition $n_{0}=n_{N+1}=0$. With this and the normalization condition (4) it is easy to see that Eq. (25) reduces to Eq. (18) for $N=2$.

Equation (25) yields real solutions for the point-to-point potential differences $V_{k+1}-V_{k}$ as long the terms under the square roots remain positive. This immediately leads to the following constraint on the time-dependent lattice density, which is a generalization of Eq. (19):

$$
\left|S_{k}\right|<\frac{\sqrt{n_{k} n_{k+1}}}{a^{2}}, \quad k=1, \ldots, N-1 .
$$

This condition represents a much tighter and more specific criterion for the $V$-representability of a given time-dependent lattice density than the upper bound (10).

Let us compare the two criteria for the same oscillating density function (21), with $A_{1}=0.77$ and $A_{2}=0.27$, that was studied in Sec. IV. We consider $N$-point lattices with grid

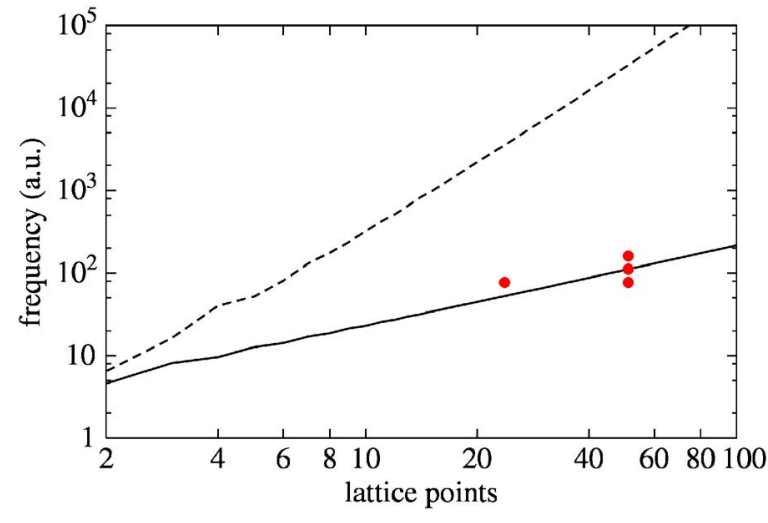

FIG. 3. (Color online) Full line: critical frequency at which the timedependent density (21) becomes non-VR on an $N$-point lattice. Dashed line: frequency at which the density would violate the upper bound (10). The dots refer to the cases studied in Fig. 4.

spacing $a=1 /(N+1)$ and lattice points at $x_{j}=j a-1 / 2, j$ $=1, \ldots, N$, and we normalize the discretized density using Eq. (4).

The full line in Fig. 3 shows the critical frequency at which the time-dependent density becomes non-VR on at least one point on an $N$-point lattice, according to Eq. (26). By comparison, the dashed line shows the frequency at which the same time-dependent density would start to violate the upper bound (10). One finds that the former increases linearly with the number of lattice points $N$, whereas the latter grows as $N^{3}$. This drastically different behavior is not surprising: the absolute upper bound (10) corresponds to the extreme limit where the density is completely concentrated on three lattice points only. For $N<4$, this gives a quite good agreement as seen in Fig. 3, but the more points one adds to the lattice, the less this extreme scenario applies to the density that we actually consider here.

Figure 4 gives further evidence of the breakdown of $V$-representability. We plot the time-dependent force associated with the potential differences (25),

$$
F_{k+1 / 2}=-\left(V_{k+1}-V_{k}\right) / a,
$$

which represents a discretized version of the continuum expression $F(x)=-\partial V(x) / \partial x$. At $N=50$ and $\omega=80 \pi$ the density (21) is VR, as can be seen from Fig. 3 (the dot below the full line). The forces $F_{k+1 / 2}$ are therefore well behaved, i.e., they exist and are finite everywhere on the lattice at all times. If we keep the number of lattice points fixed but increase the frequency, we cross the line of critical frequencies at $\omega$ $=109 \pi: V$-representability breaks down and there are regions on the lattice and times during the cycle where no force exists. A similar breakdown happens for fixed frequency if the number of lattice points is reduced.

\section{DISCUSSION}

From the above analysis of one-electron lattice densities, we can draw a number of conclusions about noninteracting $V$-representability in TDDFT.

Continuum limit. Condition (26), as well as the upper limit (10) become more and more easily satisfied as the grid spacing $a$ decreases. This is also evident from Fig. 3. Thus, 


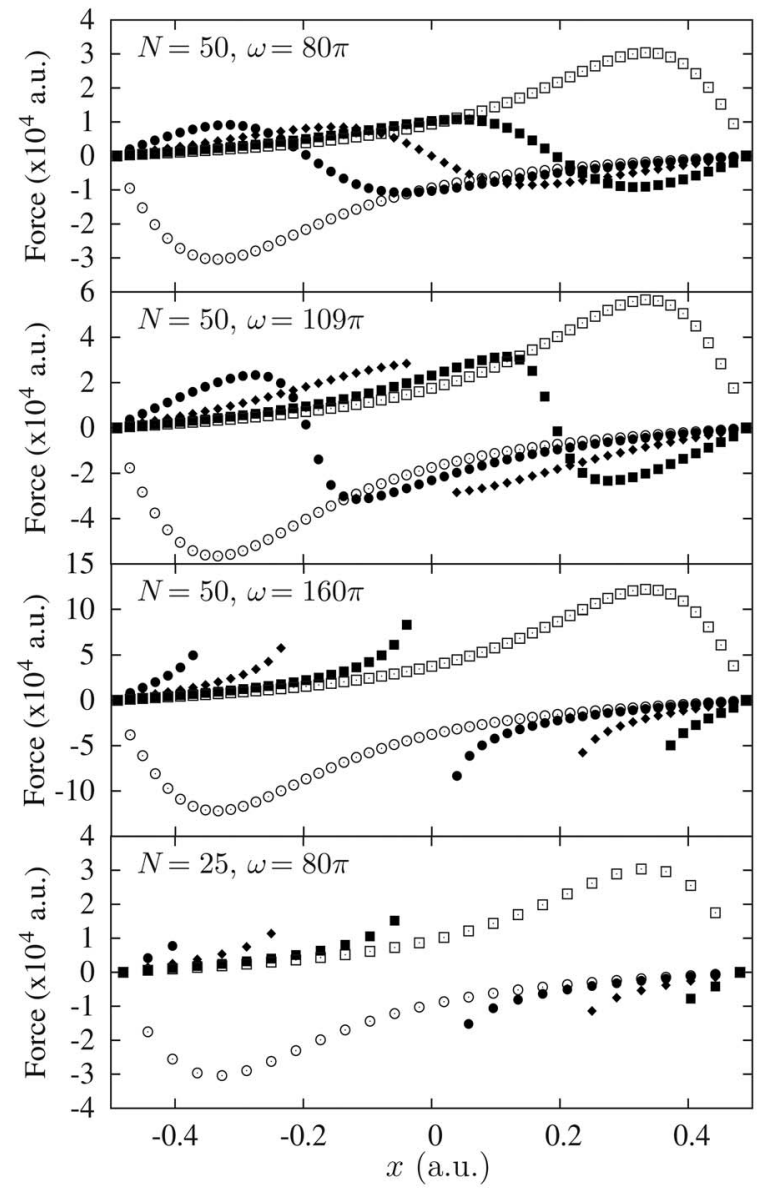

FIG. 4. Snapshots of the time-dependent force (27) generating the density (21) for $t=0$ (open circles), $T / 8$ (full circles), $T / 4$ (diamonds), $3 T / 8$ (full squares), and $T / 2$ (empty squares). Top panel: force exists everywhere at $N=50$ and $\omega=80 \pi, n(x, t)$ is lattice-VR. Other panels: $V$-representability breaks down for increasing $\omega$ at fixed $N$ or decreasing $N$ at fixed $\omega$.

in the continuum limit $a \rightarrow 0$, which corresponds to a lattice whose distribution of grid points is infinitely dense, all timedependent single-particle densities become VR.

There are various ways to see how time-dependent $V$-representability emerges in the continuum limit. First of all, as discussed in Sec. III, the continuity equation imposes upper bounds on the rate of change of lattice densities, which also limits the possible lattice currents. Overstepping these bounds violates the continuity equation, which breaks a crucial link in the chains of arguments that constitute the RG proof and the vL construction. In the $a \rightarrow 0$ limit, the continuity equation can be satisfied for any density, thus enabling the fundamental proofs of TDDFT which establish the oneon-one mapping between densities and potentials.

Another way of looking at things is to consider the energy eigenvalue spectrum associated with a particular lattice. Each excitation energy can be associated with a resonant electronic eigenmode, i.e., a free (undriven) oscillation of arbitrary amplitude. If the time dependence of a given lattice density is close to that of one of the lattice eigenmodes, or a superposition thereof, then it is possible to find an external time-dependent driving potential which gives rise to this density. On the other hand, if the given time-dependent density changes much more rapidly than any available lattice mode, then no external potential exists which is capable of inducing these rapid changes. This was clearly demonstrated in Sec. IV for the two-point lattice.

Ultimately, it is the time-energy uncertainty relation $\Delta t \Delta E \gtrsim \hbar$ which governs how fast a quantum state can change in time. ${ }^{19}$ Here $\Delta E$ indicates a spectral measure of the initial Hamiltonian of the system, such as its "energy spread." Consider a one-dimensional $N$-point lattice with grid spacing $a$ and uniform (constant) potential. There are $N$ eigenvalues $\varepsilon_{n}, n=1, \ldots, N$, of the static Schrödinger equation, and $N-1$ eigenmodes with frequency $\omega_{n}=\varepsilon_{n}-\varepsilon_{1}$. The dynamical range or energy spread of this lattice is given by the difference of the highest and the lowest eigenvalue, which scales as $1 / a^{2}$. According to the uncertainty relation, the range of possible response times of the system decreases at the same rate. Thus, the smaller the grid spacing, the more rapidly the system can change in time, and the wider the range of time-dependent $V$-representability.

Complex potentials. It may be of interest to observe that non-VR lattice densities can still be associated with external potentials. However, these potentials have to be complex and are therefore non-Hermitian. Complex potentials are a wellknown tool to generate absorbing boundary conditions for quantum dynamics simulations on finite grids. ${ }^{20}$ However, complex time-dependent potentials can also be constructed to be norm conserving, which means that the resulting time evolution is unitary. In general, such non-Hermitian Hamiltonians satisfy the physical condition of space-time reflection $(\mathcal{P} \mathcal{T})$ symmetry, as discussed in detail by Bender et al. ${ }^{21}$

In the Appendix we explicitly show how to construct complex time-dependent lattice potentials from given densities. The physical mechanism by which a complex potential reproduces a non-VR density is not by "driving" the density from one lattice point to another, as a real potential would try to do. Rather, complex potentials introduce local particle sources and sinks. A time-dependent density that moves rapidly between two lattice points can thus be viewed as being "destroyed" on the first point and "recreated" on the second point. There is in principle no limit as to how fast such processes can take place. ${ }^{22}$ This is also reflected in the fact that the continuity equations (2) and (3) are modified for complex potentials to include extra source terms.

The mapping between real densities (a single variable) and complex potentials (two variables: real and imaginary parts) obviously cannot be unique, as shown in the two-point lattice example in Appendix. It may be possible to restore the uniqueness of the mapping by including the phase information of the wave function, or equivalently by using the current as an additional variable.

Many-electron systems. In this paper, we have only studied the case of a single electron in detail. However, the essential arguments for and against noninteracting $V$-representability on lattices, see Sec. III, can be carried over to the case of many electrons. In the time-dependent Kohn-Sham (TDKS) scheme, the continuity equation holds for each individual Kohn-Sham orbital, and it is immediately seen that this can be violated on a finite lattice. The upper bound (10) can be generalized to the case of several electrons in a straightforward manner. It is thus clear that the time- 
dependent $V$-representability for many-electron system is only guaranteed in continuous space, and not on lattices.

It is possible to invert the TDKS equation for two electrons in a doubly occupied orbital, which was recently done for Hooke's atom ${ }^{23-25}$ and two-dimensional quantum strips. ${ }^{26}$ However, for more than two electrons the construction of the potential in terms of the density can no longer be carried out analytically [such as in Eq. (25)], but only numerically.

Ensemble-VR densities. In static DFT, examples of non-VR densities were first constructed by Levy. ${ }^{27}$ The conundrum was soon resolved by recognizing that such densities can come from ensembles of degenerate states. This finally led to the modern view of $V$-representability in DFT in terms of pure- and ensemble-state VR densities, see the discussion in Sec. II.

The role played by degeneracies in TDDFT has so far not been studied in much detail; the situation is much more complex than in static DFT. However, it is highly unlikely that the absence of $V$-representability on lattices can be cured by the same trick that worked in static DFT, that is, by representing non-VR densities via ensembles of degenerate states. The simple reason is that, unlike in static DFT, non-VR time-dependent densities occur already for oneelectron systems. Furthermore, the physical origins of non- $V$-representability in TDDFT are fundamentally tied to the dynamics (such as the continuity equation and how fast quantum states can change-see the above discussion).

Practical consequences. In Ref. 8 it was argued that $V_{\mathrm{XC}}$ is likely to be an extremely sensitive functional of the timedependent density, especially in strongly time-dependent problems, due to the possible occurrence of instabilities and breakdown of the density-on-potential mapping on lattices. Indeed, $V_{\mathrm{XC}}$ is defined as a functional of the time-dependent density on the domain of densities that are noninteracting $\mathrm{VR}$, and is therefore undefined for non-VR densities. However, does this have any practical consequences for TDDFT?

$V_{\mathrm{XC}}$ needs to "know" whether a time-dependent lattice density is noninteracting VR or not. Continuum-space XC functionals are not required to have this property, since all time-dependent continuum densities are noninteracting VR by the vL construction ${ }^{16}$ (as long as the initial state is VR). Therefore, the exact or any approximate $V_{\mathrm{XC}}$ from continuum TDDFT, if used in a lattice TDKS calculation, remains well defined (real and finite) even if evaluated with non-VR lattice densities. To deal properly and consistently with non-VR lattice densities, $V_{\mathrm{XC}}$ must be lattice dependent, i.e., it needs to be constructed for many-body systems on specific lattices.

Let us now assume that we have the exact $V_{\mathrm{XC}}$ for a specific lattice system, and we want to carry out a selfconsistent TDKS calculation using an iterative procedure such as discussed in Ref. 28. The first iteration step involves evaluating $V_{\mathrm{XC}}$ with a time-dependent trial density. If this trial density turns out to be non-lattice-VR, then the TDKS calculation breaks down. If, on the other hand, the trial density is lattice-VR, then each subsequent iteration produces only lattice-VR densities, and the TDKS calculation will be well behaved.

Problems could still arise if $V_{\mathrm{XC}}$ were extremely sensitive within the VR region: for example, if it fluctuated wildly for VR lattice densities that are close to the non-VR region. However, the examples which we have studied here indicate that this is unlikely to be the case. Figure 4 shows that the force, and hence the potential itself, remains finite and well behaved as long as the density is VR, and otherwise simply ceases to exist.

Finally, it is an obvious statement that in the usual applications of TDDFT the density follows from the potential, not the other way round, and therefore remains VR by definition. Furthermore, we have seen that non- $V$-representability becomes increasingly rare in the space of time-dependent lattice densities if the grid spacing is made smaller and smaller by adding more and more lattice points. We may therefore conclude that the fact that the density-on-potential mapping on lattices can break down has no practical consequences for TDDFT.

This work was supported by NSF Grant No. DMR0553485 and by Research Corporation. We thank Giovanni Vignale, Ilya Tokatly, and Klaus Capelle for helpful comments.

\section{APPENDIX: COMPLEX POTENTIALS}

Let us first revisit the derivation of Sec. IV for the twopoint lattice, but now admitting a time-dependent potential with nonvanishing imaginary part. We impose norm conservation,

$$
0=\dot{n}_{1}+\dot{n}_{2}=\left(\psi_{1}^{*} \dot{\psi}_{1}+\psi_{1} \dot{\psi}_{1}^{*}\right)+\left(\psi_{2}^{*} \dot{\psi}_{2}+\psi_{2} \dot{\psi}_{2}^{*}\right) .
$$

Making use of the TDSE for $\psi_{1}$ and $\psi_{2}$, this leads to the following condition for the imaginary parts of the potentials on points 1 and 2 :

$$
n_{1} \mathfrak{I} V_{1}+n_{2} \mathfrak{I} V_{2}=0
$$

From Eq. (15) we obtain

$$
\alpha_{1}-\alpha_{2}=\sin ^{-1}\left(\frac{a^{2} \dot{n}_{1}}{\sqrt{n_{1} n_{2}}}-2 a^{2} \sqrt{\frac{n_{1}}{n_{2}}} \Im V_{1}\right) \text {. }
$$

Taking the time derivative of this and plugging it into Eq. (17) give, after some algebra,

$$
\begin{aligned}
\Re V_{2}- & \Re V_{1} \\
= & \frac{a^{2}}{\sqrt{n_{1} n_{2}-a^{4}\left(\dot{n}_{1}-2 n_{1} \mathfrak{I} V_{1}\right)^{2}}} \\
& \times\left\{\ddot{n}_{1}+\frac{\dot{n}_{1}^{2}\left(n_{1}-n_{2}\right)}{2 n_{1} n_{2}}-\frac{\dot{n}_{1}}{n_{2}} \mathfrak{I} V_{1}-2 n_{1} \mathfrak{I} \dot{V}_{1}\right\} \\
& +\frac{n_{1}-n_{2}}{2 a^{2} n_{1} n_{2}} \sqrt{n_{1} n_{2}-a^{4}\left(\dot{n}_{1}-2 n_{1} \mathfrak{I} V_{1}\right)^{2}},
\end{aligned}
$$

which is a generalization of Eq. (18) for nonvanishing imaginary part of the potential. We saw in Sec. VI that the density is non-VR if the argument $n_{1} n_{2}-a^{4} \dot{n}_{1}^{2}$ under the square root in Eq. (18) becomes negative. If we admit complex potentials, we have the freedom to choose $\mathfrak{I} V_{1}$ in such a way that 


$$
n_{1} n_{2}-a^{4}\left(\dot{n}_{1}-2 n_{1} \Im V_{1}\right)^{2}>0 .
$$

This determines $\mathfrak{R} V_{2}-\mathfrak{R} V_{1}$ and $\mathfrak{I} V_{2}$ via Eqs. (A2) and (A4). Clearly, $\mathfrak{I} V_{1}$ is not unique, but each choice for $\mathfrak{I} V_{1}$ which satisfies condition (A5) will reproduce the given timedependent density via the lattice-TDSE (13).

The arguments presented here for the two-point lattice can be extended for the $N$-point lattice in a straightforward manner. Generalizing Eq. (A2), we find

$$
\sum_{j=1}^{N} n_{j} \mathfrak{I} V_{j}=0
$$

as a necessary condition that the complex lattice potential is norm conserving. The point-to-point differences of the real parts of the potential, $\mathfrak{R} V_{k+1}-\mathfrak{R} V_{k}$, can then be obtained from Eq. (25) by replacing $S_{k}$ in Eq. (24) by

$$
S_{k}=\sum_{j=1}^{k}\left(\dot{n}_{j}-2 n_{j} \mathfrak{I} V_{j}\right) \text {. }
$$

We can therefore always satisfy the constraint (26) through appropriate choices of the imaginary part of the lattice potential, consistent with the norm-conservation condition (A6). As for the two-point lattice, these choices are not unique.

${ }^{1}$ Time-Dependent Density Functional Theory, Lecture Notes in Physics Vol. 706, edited by M. A. L. Marques, C. A. Ullrich, F. Nogueira, A. Rubio, K. Burke, and E. K. U. Gross (Springer, Berlin, 2006).
${ }^{2}$ E. Runge and E. K. U. Gross, Phys. Rev. Lett. 52, 997 (1984).

${ }^{3}$ P. Hohenberg and W. Kohn, Phys. Rev. 136, B864 (1964).

${ }^{4}$ W. Kohn, Rev. Mod. Phys. 71, 1253 (1999).

${ }^{5}$ H. O. Wijewardane and C. A. Ullrich, Phys. Rev. Lett. 95, 086401 (2005).

${ }^{6}$ C. A. Ullrich and I. V. Tokatly, Phys. Rev. B 73, 235102 (2006).

${ }^{7}$ G. Vignale, Phys. Rev. A 77, 062511 (2008).

${ }^{8}$ R. Baer, J. Chem. Phys. 128, 044103 (2008).

${ }^{9}$ R. M. Dreizler and E. K. U. Gross, Density-Functional Theory: An Approach to the Quantum Many-Body Problem (Springer, Berlin, 1990).

${ }^{10}$ J. Chen and M. J. Stott, Phys. Rev. A 47, 153 (1993); 44, 2816 (1991).

${ }^{11}$ W. Kohn, Phys. Rev. Lett. 51, 1596 (1983).

${ }^{12}$ J. T. Chayes, L. Chayes, and M. B. Ruskai, J. Stat. Phys. 38, 497 (1985).

${ }^{13}$ C. A. Ullrich and W. Kohn, Phys. Rev. Lett. 89, 156401 (2002).

${ }^{14}$ P. E. Lammert, J. Chem. Phys. 125, 074114 (2006).

${ }^{15}$ C. A. Ullrich, Phys. Rev. B 72, 073102 (2005).

${ }^{16}$ R. van Leeuwen, Phys. Rev. Lett. 82, 3863 (1999).

${ }^{17}$ M. Abramowitz and I. A. Stegun, Handbook of Mathematical Functions (Dover, New York, 1965).

${ }^{18}$ C. Verdozzi, e-print arXiv:0707.2317v1; (private communication).

${ }^{19}$ P. Pfeifer, Phys. Rev. Lett. 70, 3365 (1993); P. Pfeifer and J. Fröhlich, Rev. Mod. Phys. 67, 759 (1995).

${ }^{20}$ Á. Vibók and G. G. Balint-Kurti, J. Phys. Chem. 96, 8712 (1992).

${ }^{21}$ C. M. Bender, D. C. Brody, and H. F. Jones, Am. J. Phys. 71, 1095 (2003).

${ }^{22}$ C. M. Bender, D. C. Brody, H. F. Jones, and B. K. Meister, Phys. Rev. Lett. 98, 040403 (2007).

${ }^{23}$ I. D'Amico and G. Vignale, Phys. Rev. B 59, 7876 (1999).

${ }^{24}$ P. Hessler, N. T. Maitra, and K. Burke, J. Chem. Phys. 117, 72 (2002).

${ }^{25}$ A. S. de Wijn, S. Kümmel, and M. Lein, J. Comput. Phys. 226, 89 (2007).

${ }^{26}$ C. A. Ullrich, J. Chem. Phys. 125, 234108 (2006).

${ }^{27}$ M. Levy, Phys. Rev. A 26, 1200 (1982).

${ }^{28}$ H. O. Wijewardane and C. A. Ullrich, Phys. Rev. Lett. 100, 056404 (2008). 